See discussions, stats, and author profiles for this publication at: https://www.researchgate.net/publication/330880296

\title{
Improved Human-Robot Collaborative Control of Redundant Robot for Teleoperated Minimally Invasive Surgery
}

Article · February 2019

DOI: 10.1109/LRA.2019.2897145

CITATIONS

0

4 authors, including:

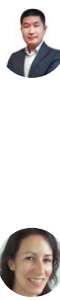

Hang Su

Politecnico di Milano

17 PUBLICATIONS 171 CITATIONS

SEE PROFILE

Elena De Momi

Politecnico di Milano

169 PUBLICATIONS 1,094 CITATIONS

SEE PROFILE

Some of the authors of this publication are also working on these related projects:

MUNDUS View project

Project ACTIVE View project
READS

67

Giancarlo Ferrigno

Politecnico di Milano

340 PUBLICATIONS 4,561 CITATIONS

SEE PROFILE 


\title{
Improved Human-Robot Collaborative Control of Redundant Robot for Teleoperated Minimally Invasive Surgery
}

\author{
Hang $\mathrm{Su}^{1}$, Chenguang Yang $^{2}$, Giancarlo Ferrigno ${ }^{1}$ and Elena De Momi ${ }^{1}$
}

\begin{abstract}
An improved human-robot collaborative control (IHRCC) scheme is proposed in teleoperated Minimally Invasive Surgery (MIS) scenario, based on a hierarchical operational space formulation of a seven degrees of freedom (DoFs) redundant robot. Redundancy is exploited to guarantee a remote center of motion (RCM) constraint and to provide a compliant behavior for the medical staff. Based on the implemented hierarchical control framework, an RCM constraint and a safe constraint are applied to the null-space motion to achieve the surgical tasks with human-robot interaction. Due to the physical interactions, safety and accuracy of the surgery may be affected. The control framework integrates adaptive compensator to enhance the accuracy of surgical tip and to maintain the RCM constraint in a decoupled way avoiding any physical interactions. The system performance is verified on a patient phantom. Compared with the methods proposed in the literature, results show that the accuracy of both RCM constraint and surgical tip are improved. The compliant swivel motion of the robot arm is also constrained in a defined area, and the interaction force on the abdominal wall become smaller.
\end{abstract}

Index Terms-Physical Human-Robot Interaction; Redundant Robots; Surgical Robotics: Laparoscopy

\section{INTRODUCTION}

$\mathbf{I}$ $\mathrm{N}$ teleoperated Minimally Invasive Surgery (MIS), the surgical tool tip must go through small abdominal incisions during the teleoperated surgery. It is important to assure that the tool does not apply big forces on the incision wall in order to prevent patient harm. Hence, each small incision produces a kinematic constraint in the remote site, known as Remote Center of Motion (RCM) [1], as shown in Fig. 1.

Respecting the RCM constraint without any loss of accuracy of the surgical tip has always been a challenging task and has attracted research interests in recent years. In general, the RCM constraint can be active or passive. In the passive way, the constraint is mechanically maintained by utilizing spherical

Manuscript received: September 7, 2018; Revised December 2, 2018; Accepted December 31, 2018.

This paper was recommended for publication by Editor Allison M. Okamura, upon evaluation of the Associate Editor and Reviewers' comments. *This work was supported by the European Unions Horizon 2020 research and innovation program under SMARTsurg project grant agreement No. 732515. This work was partially supported by Engineering and Physical Sciences Research Council (EPSRC) under Grant EP/S001913/1.

${ }^{1} \mathrm{Hang} \mathrm{Su}$, Giancarlo Ferrigno and Elena De Momi are with the Department of Electronics, Information and Bioengineering, Politecnico di Milano, 20133, Milan, Italy. hang.su, giancarlo.ferrigno, elena.demomi@polimi.it

${ }^{2}$ Chenguang Yang is with Bristol Robotics Laboratory, University of the West of England, Bristol, BS16 1QY, UK. cyang@ieee.org

Digital Object Identifier (DOI): see top of this page. mechanisms or by a dual-parallelograms design, and its joints is passively controlled to guarantee that no big forces on the abdominal wall. The active way achieves the RCM constraint directly with a software controller [2].

Parallel-type robots, such as the AESOP manipulator and the da Vinci surgical system, are specifically designed to passively achieve the motion [3]. Whereas, open-chained manipulators, such as the DLR MIRO robots, usually adopt the motion controller to maintain the RCM constraint [4]. Compared with the passive way, the active way is more popular in nonclinical research [2][5][6], since it provides a lot of benefits, like economic feasibility and task-space flexibility. Ortmaier et al. implemented an inverse kinematic control to achieve RCM constraint motion, preventing any force exerted on the trocar [7] without using high-cost mechanisms to achieve the constraint. In our previous work [6][8], we solved the RCM constraint by trajectory planning in the Cartesian space and implemented an impedance controller to control it in an active way.

High contact forces may emerge in case of rigid interaction[9][10], which can be dangerous for the surgical operation. Impedance control provides an efficient way to avoid rigid interaction and force overshoots. However, most of the published literature did not consider the soft physical interaction due to the RCM constraint and did not analyze the force exerted on the abdominal wall.

Additional tasks can be achieved by exploiting the redundancy of the robots, for example, obstacle avoidance [11] and human-like behavior [12]. It is effective to utilize the redundancy to maintain the active RCM constraint. Sandoval et $a l$. proposed an improved dynamic control approach to utilize the redundancy for RCM constraint [5]. In our previous work, the redundancy was utilized to provide flexible workspace [6][13] and an adaptive decoupling controller [14] was applied to achieve the RCM constraint. Furthermore, we utilize the redundancy to provide flexible workspace with compliant motion behavior for the nurse or surgeon to assist physicians [6][8]. In our previous work, we proposed a safety enhanced controller to apply a constrained compliant behavior on the robot's arm, avoiding the loss of accuracy with human-robot interaction, and introduced a direct fuzzy adaptive approximation [15] to compensate the disturbance from human-robot interaction [8].

However, previous strategies solved the RCM constraint with trajectory planning in the task space without decoupling, which influences the accuracy of surgical tip. Dietrich et al. introduced a Hierarchical Operational Space combining mul- 
tiple null-space controllers and proved its stability [16][17], making it possible to solve the RCM constraint and the compliant swivel behavior with hierarchical null-spaces in a single controller.

In this paper, we utilize the hierarchical operational space formulation [17] to achieve whole-body impedance control of a 7-DoF serial robot. The surgical task implementation in [8] is improved with our novel strategy by achieving RCM constraint in the $1^{\text {st }}$ Null-space of the Jacobian matrix of surgical tip. We add a safety-enhanced compliant arm behavior in the $2^{\text {nd }}$ Nullspace, which is of the Jacobian matrix for RCM constraint. Furthermore, we introduce a decoupled fuzzy compensator to compensate the disturbance, maintaining the RCM constraint and improving the surgical accuracy, achieving human-robot collaborative control in teleoperated surgery. Experiments are performed in a lab setup environment using the 7 degrees of freedom (DoFs) LWR4+ (KUKA, Germany) and Sigma 7 (Force dimension, Switzerland) to validate the proposed control method.

This paper is organized as follows: Firstly, the surgical scenario and the proposed Methodology is described in Section II. The experimental demonstration is explained in Section III. At the end, in Section IV, a further discussion and conclusions are drawn.

\section{Methodology}

\section{A. Prior work}

1) Modeling of the Serial Robot: The dynamic model of an $n$ DoFs serial manipulator can be expressed as [18]:

$$
M(q) \ddot{q}+C(q, \dot{q}) \dot{q}+g(q)-\tau_{e}=\tau_{C}
$$

where $\boldsymbol{q} \in R^{n}$ is the joint vector, $\boldsymbol{M}(\boldsymbol{q}) \in R^{n \times n}$ is the inertia matrix, $\boldsymbol{C}(\boldsymbol{q}, \dot{\boldsymbol{q}}) \in R^{n \times n}$ is the matrix of Coriolis force and Centrifugal effects, and the corresponding gravity vector is $\boldsymbol{g}(\boldsymbol{q}) \in R^{n} . \tau_{C} \in R^{n}$ and $\tau_{\boldsymbol{e}} \in R^{n}$ denote the control torques and the external torque, respectively. The serial manipulator is assumed to be far away from singularity for simplification. It is also assumed that the pseudo-inverse of the Jacobian matrix $\boldsymbol{J}_{T}(\boldsymbol{q}) \in R^{m \times n}$ from the base to tip exists.

The above generalized formulation in the task space can be rewritten as follows [19][20]:

$$
M_{X}(X) \ddot{X}+H_{X}(X, \dot{X}) \dot{X}+J_{T}^{-T}(q) g(q)-F_{e T}=F
$$

where $\boldsymbol{X} \in R^{m}$ is the task space coordinates vector and $\dot{X} \in R^{m}$ is the actual Cartesian velocity,

$$
\begin{aligned}
M_{X}(X) & =J_{T}^{-T} M(q) J_{T}^{-1} \\
H_{X}(X, \dot{X}) & =J_{T}^{-T}\left[C(q, \dot{q})-M(q) J^{-1} \dot{J}_{T}\right] J_{T}^{-1} \\
F_{e T} & =J_{T}^{-T} \tau_{e}
\end{aligned}
$$

The matrix $\boldsymbol{M}_{\boldsymbol{X}}(\boldsymbol{X}) \in R^{m \times m}$ is the Cartesian inertia, $\boldsymbol{H}_{\boldsymbol{X}}(\boldsymbol{X}, \dot{\boldsymbol{X}}) \in$ $R^{m \times m}$ is the Cartesian Coriolis force effects, $\boldsymbol{F}_{\boldsymbol{e} \boldsymbol{T}} \in R^{m}$ is the external force vector in task space, with

$$
\exists \beta \in R,\left\|\boldsymbol{F}_{\boldsymbol{e} T}\right\| \leq \beta, \forall t \geq 0
$$

Property 2.1: The Cartesian inertia matrix $\boldsymbol{M}_{\boldsymbol{X}}(\boldsymbol{X})$, as defined in (3), is bounded as:

$$
\lambda_{1}\|\boldsymbol{A}\| \leq \boldsymbol{A}^{\boldsymbol{T}} \boldsymbol{M}_{\boldsymbol{X}}(\boldsymbol{X}) \boldsymbol{A} \leq \lambda_{2}\|\boldsymbol{A}\|, \forall \boldsymbol{A}, \boldsymbol{X} \in R^{m}
$$

and it is positive and symmetric, where $\lambda_{1}>0 \in R$ and $\lambda_{2}>$ $0 \in R$.

Property 2.2: The Coriolis and Centrifugal force matrix $\boldsymbol{H}_{\boldsymbol{X}}(\boldsymbol{X}, \dot{\boldsymbol{X}})$ and the time derivative of the Cartesian inertia, $M_{X}(X)$, satisfy

$$
A^{T}\left[\dot{M}_{X}(X)-2 H_{X}(X, \dot{X})\right] A=0, \forall A, q, \dot{q} \in R^{n}
$$

\section{2) Hierarchical Operational Space Formulation:}

$$
\dot{X}=J_{T}(q) \dot{q}
$$

Since the number of DoFs is greater than $m=3$, the redundancy could be utilized to accomplish additional tasks. The extended Jacobian method [21] is introduced to enrich the task space, including both the end-effector and the null-space kinematic coordination. The corresponding control law of the extended dynamical formulation can be written as [20][22]:

$$
\begin{array}{r}
F_{T}=M_{X} \ddot{X}_{d}+H_{X} \dot{X}_{d}-K_{X}\left(X-X_{d}\right)-D_{X}\left(\dot{X}-\dot{X}_{d}\right) \\
F_{N_{1}}=-K_{N 1}\left(N_{1}-N_{1 d}\right)-D_{N 1}\left(\dot{N}_{1}-\dot{N}_{1 d}\right) \\
F_{N_{2}}=-K_{N 2}\left(N_{2}-N_{2 d}\right)-D_{N 2}\left(\dot{N}_{2}-\dot{N}_{2 d}\right)
\end{array}
$$

where $\boldsymbol{F}_{\boldsymbol{T}} \in R^{3}$ is the active force in task space, $\boldsymbol{F}_{\boldsymbol{N} \mathbf{1}}, \boldsymbol{F}_{\boldsymbol{N} \mathbf{2}}$ are the forces applied on the null-space kinematics, $N_{1}, N_{2}$ are the actual null-space coordinates vectors, and $\boldsymbol{N}_{\mathbf{1 d}}, \boldsymbol{N}_{\mathbf{2} d}$ are the corresponding desired null-space coordinates. Finally, the force to achieve RCM can be mapped into joint torques using the controller $\left(\tau_{N} \in R^{n}\right)$, which is defined as:

$$
\tau_{N}=\left(I-J_{T}^{T}(q) J_{T}(q)_{M}^{+}\right) J_{N}^{T} F_{N}
$$

where $\boldsymbol{J}_{N} \in 3^{n-m}$ is the Jacobian matrix from the robot base to the null-space kinematics, and $\boldsymbol{J}_{\boldsymbol{T}}(\boldsymbol{q})_{M}^{+}$is the inertia-weighted pseudo-inverse matrix [19]:

$$
J_{T}(q)_{M}^{+}=\left(J_{T}(q) M(q)^{-1} J_{T}(q)^{T}\right)^{-1} J_{T}(q) M(q)^{-1}
$$

Fig. 2 shows the hierarchical control architecture and its stability analysis are verified in [20][22].

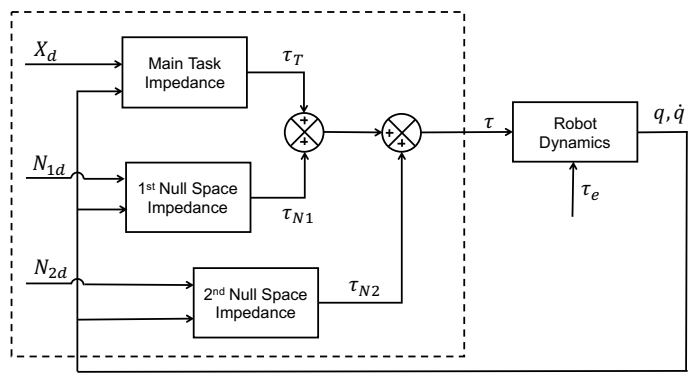

Fig. 2. Block diagram of the hierarchical control architecture.

3) Teleoperated Surgical Task Implementation: The interaction force $\boldsymbol{F}_{\boldsymbol{e}}$, shown in Fig. 3, between the surgical tool shaft and the abdominal wall, should be minimized during the teleoperated task to avoid increasing the invasive wound. To drive the end-effector position $\left(X \in R^{3}\right)$ reaching the desired position $\left(\boldsymbol{X}_{\boldsymbol{r}} \in R^{3}\right)$ from the master, an interpolation method is introduced in [23]:

$$
X_{d}=-k_{0}\left(X-{ }^{s} T_{m} X_{r}\right)+{ }^{s} T_{m} \dot{X}_{r}
$$




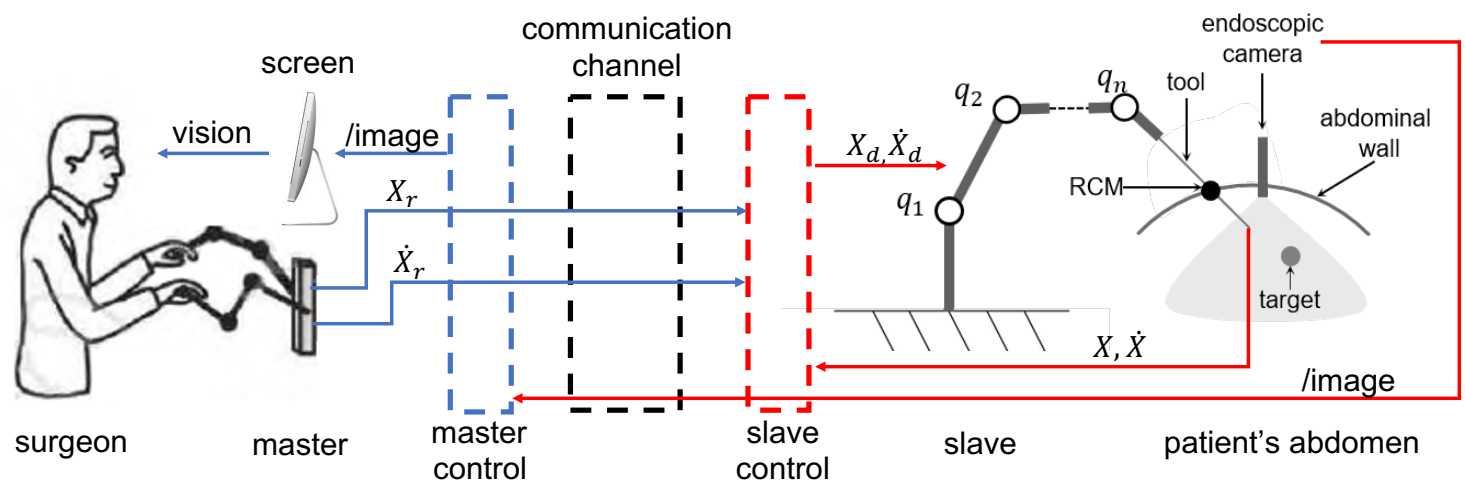

Fig. 1. Typical teleoperated Minimally Invasive Surgery structure. $\dot{X}_{r}, \boldsymbol{X}_{r} \in R^{m}$ denote the reference Cartesian velocity and position in the master frame, respectively. $\boldsymbol{X}, \dot{\boldsymbol{X}} \in R^{m}$ represent the actual Cartesian position and velocity in the slave frame, respectively. $\dot{\boldsymbol{X}}_{\boldsymbol{d}}, \boldsymbol{X}_{\boldsymbol{d}} \in R^{m}$ are the desired Cartesian velocity and position in the slave frame, respectively.

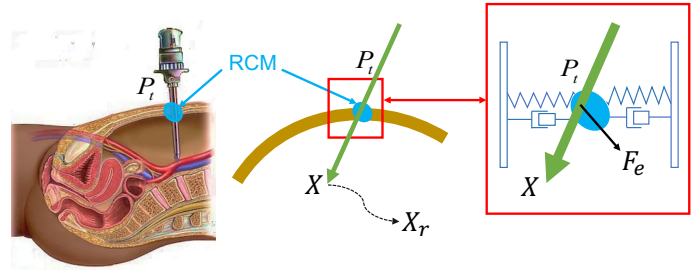

Fig. 3. Minimally invasive surgery scene: $P_{t}$ is the trocar position, representing the RCM Constraint. During the surgery, the tool shaft must go through the trocar. The tool tip should follow the reference trajectory in the cavity of the abdomen. $F_{e}$ is the force exerted on the abdominal wall by the tool shaft.

where $k_{0}>0$ is a constant and ${ }^{\boldsymbol{s}} \boldsymbol{T}_{\boldsymbol{m}}$ is the transformation matrix between the master frame and the slave frame. The compliance control term, $\tau_{T} \in R^{7}$, defined in our previous work [6][8], is introduced to achieve impedance control of the surgical tip.

4) Safety-enhanced Compliant Arm Behavior: The tool tip was moved to perform surgical tasks respecting the RCM constraint. The swivel angle, $\psi \in R$, of the robot arm, defined in [24], was left as kinematic redundancy. In our previous work [6][8], a safe swivel motion constraint $\left[\psi_{\min f}, \psi_{\max f}\right]$ is defined, which is assumed to depend on the actual situation, and a virtual force $\boldsymbol{F}_{\psi}$ is defined to prevent the swivel motion from exceeding the boundary.

\section{B. Control System Development}

A hierarchical operational space formulation is introduced to integrate the teleoperated surgical task implementation and the safety-enhanced compliant arm behavior in our previous work [6][13]. The surgical task implementation in [8] is improved by achieving RCM constraint and the safety-enhanced compliant arm behavior in two separate Null-space of the Jacobian matrix of the robot tool tip and wrist. A novel decoupled adaptive fuzzy compensation to is introduced to enhance both the accuracy for the surgical tip and the RCM constraint. Details are listed as follows:

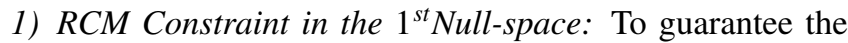
RCM constraint, a distance error $d=\left\|\left(\boldsymbol{P}_{t}-\boldsymbol{X}\right) \times \hat{\boldsymbol{u}}_{c}\right\| \in R$ is defined in Fig. 4, where $\hat{\boldsymbol{u}}_{c} \in R^{3}$ is the direction vector of the

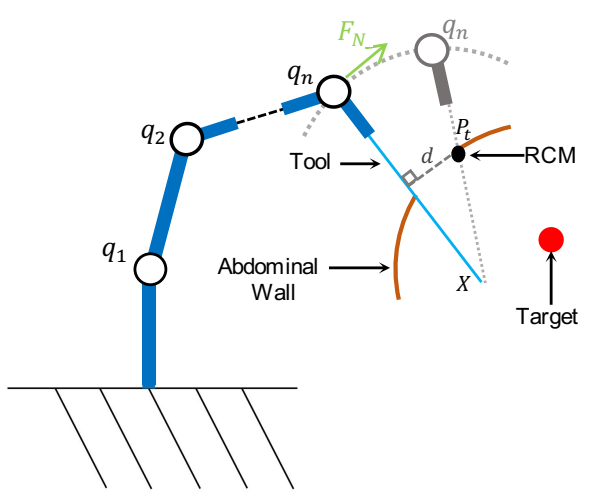

Fig. 4. Achieve RCM constraint with null-space controller: $d$ is the distance between trocar position $\left(\boldsymbol{P}_{\boldsymbol{t}}\right)$ and tool shaft (the small incision is zoomed for a better understanding). The actual tool-tip position $\boldsymbol{X}$ is controlled by teleoperation to reach the target in patient's abdomen, $\boldsymbol{F}_{\boldsymbol{N}}$ is the virtual force applied to the robot wrist to rotate the surgical tip.

surgical tool shaft. To satisfy the constraint without having any influence on the main surgical task, a safer solution is to drive the "wrist" $\left(q_{n}\right)$ joint tracking a circular movement around the end-effector from the actual position, $N_{1}$, to the desired position, $\boldsymbol{N}_{\mathbf{1} \boldsymbol{d}}$, until the tool shaft passes through the trocar position, $\boldsymbol{P}_{t}$, as shown in Fig. 4. Accordingly, the gray joint $q_{n}$ is viewed as the desired position $\left(\boldsymbol{N}_{\mathbf{1} d}\right)$ of the last joint. Hence, an RCM controller can be introduced as:

$$
F_{N}=-K_{N} e_{N 1}-D_{N} \dot{e}_{N 1}
$$

where $\boldsymbol{D}_{N}, \boldsymbol{K}_{N} \in R^{3}$ are the corresponding damping and stiffness matrix, $\dot{N}_{1}$ is the actual velocity of the "wrist" and $e_{N}=N_{1}-N_{1 d}$ is the tracking error. Since $\left\|e_{N}\right\|$ is proportional to the RCM constraint error $\boldsymbol{d}$ : $\boldsymbol{d}=\lambda\left\|\boldsymbol{e}_{\boldsymbol{N} 1}\right\|, 0<\lambda \leq 1$. Hence, $\boldsymbol{e}_{N \mathbf{1}}=\left(\boldsymbol{P}_{\boldsymbol{t}}-\boldsymbol{X}\right) \times \hat{\boldsymbol{u}}_{c} / \lambda$. The first null-space controller can be introduced as:

$$
\tau_{N_{11}}=\left(I-J_{T}^{T}(q) J_{T}(q)_{M}^{+}\right) J_{W}^{T} F_{N}
$$

where $\boldsymbol{J}_{W}(\boldsymbol{q}) \in R^{3 \times 5}$ represents the Jacobian matrix from the robot base to the robot wrist. In general, $\lambda$ varies according to the tip position during surgery. In this paper, we choose $\lambda$ 
as a constant, but we resolve the time-varying system with a fuzzy compensator in the following steps.

2) Constrained Compliant Behavior in the $2^{\text {nd }}$ Null-space: The constrained compliant behavior, introduced in our previous work [6][8], is defined as:

$$
\tau_{N_{2}}=\left(I-J_{W}^{T}(q) J_{W}(q)_{M}^{+}\right) J_{E}^{T} F_{\psi}
$$

where $J_{E}(q) \in R^{3 \times 3}$ is the Jacobian matrix of the robot elbow. Virtual force $\boldsymbol{F}_{\psi}$ is utilized to constrain the compliant swivel motion in a defined safe range, shown in Fig. 5.

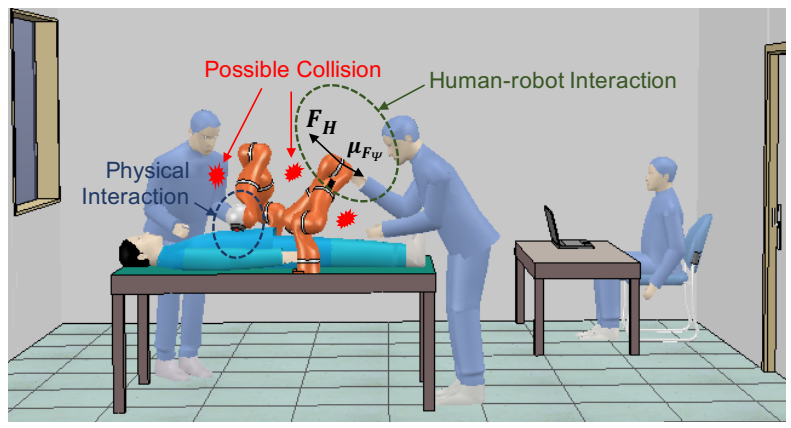

Fig. 5. Constraint compliant arm behavior: Virtual force $\boldsymbol{F}_{\psi}$ is introduced to limit the compliant motion range when there is a collision in the workspace.

3) Decoupled adaptive fuzzy compensation for uncertain disturbances with physical interaction: The surgical tool shaft and the patient's abdominal wall interact with each other during the surgery, as shown in Fig. 3. It should also be mentioned that tissue characteristic discrepancies exist and vary between different patients [25][26]. The dynamics of $\boldsymbol{F}_{\boldsymbol{e}}$ is unknown and nonlinear in a real clinical application and has been introduced for analysis. Furthermore, the hand force, $\boldsymbol{F}_{\boldsymbol{H}}$, shown in Fig. 7, applied on the robot arm [6][8] is also uncertain and time-varying. It is known that $\boldsymbol{F}_{\boldsymbol{e}}$ and $\boldsymbol{F}_{\boldsymbol{H}}$ can be decomposed into two components influencing the accuracy of the RCM constraint and the surgical tip, separately. The execution of an accurate task could be viewed as controlling the tip despite external disturbances.

A nonlinear disturbance can be approximated with $\boldsymbol{f}(\boldsymbol{Z})$ : $R^{n} \rightarrow R^{m}$ as $\boldsymbol{f}(\boldsymbol{Z})=\boldsymbol{\theta}^{\boldsymbol{T}} \boldsymbol{S}(\boldsymbol{Z})+\varepsilon(\boldsymbol{Z})$, where $\boldsymbol{\theta} \in R^{l}$ is the adaptable weight, the vector $\boldsymbol{Z}=\left[z_{1}, z_{2}, \ldots, z_{n}\right] \in R^{n}$ is the input vector of the approximation, $\boldsymbol{S}(\boldsymbol{Z}) \in R^{l}$ is the basis function vector, and $\varepsilon \in R$ is the approximation error, meeting the condition: $\exists \bar{\varepsilon} \in R^{+},|\varepsilon(\boldsymbol{Z})| \leq \bar{\varepsilon}, \forall \boldsymbol{Z} \in \boldsymbol{\Omega}_{Z}$. In this paper, the adaptive rule [27] is chosen by

$$
\xi_{j}=\frac{\prod_{i=1}^{n} \mu_{A_{i}^{l}\left(z_{i}\right)}}{\sum_{j=1}^{m} \prod_{i=1}^{n} \mu_{A_{i}^{l}\left(z_{i}\right)}}, j=1, \ldots, m
$$

The weight parameters $\Theta$ is adjusted with $\dot{\Theta}=\left[\dot{\boldsymbol{\theta}_{1}}, \dot{\boldsymbol{\theta}_{2}}, \ldots, \dot{\boldsymbol{\theta}_{m}}\right] \in$ $R^{m \times l}, \dot{\boldsymbol{\theta}}_{\boldsymbol{i}} \in R^{l}, i=1,2, \ldots, m$ :

$$
\dot{\theta}_{i}=\left\{\begin{array}{rr}
\gamma e_{i} P \xi^{T}(Z), & \left\|\theta_{i}\right\|<M_{\theta_{i}} \text { or }\left(\left\|\theta_{i}\right\| \geq M_{\theta_{i}}\right. \\
& \left.\& \gamma E P \xi^{T}(Z)<0\right) . \\
\Gamma(Z), & \left\|\theta_{i}\right\| \geq M_{\theta_{i}} \& \gamma e_{i} P \xi^{T}(Z) \geq 0 .
\end{array}\right.
$$

where $\boldsymbol{\Gamma}(\boldsymbol{Z})=\boldsymbol{\gamma} \boldsymbol{e}_{i} \boldsymbol{P}\left(\mathbf{1}-\frac{\boldsymbol{\theta}_{i} \boldsymbol{\theta}_{i}^{T}}{\left\|\boldsymbol{\theta}_{i}\right\|^{2}}\right) \boldsymbol{\xi}^{\boldsymbol{T}}(\boldsymbol{Z})$, and $\boldsymbol{\gamma} \in R^{m \times m}$ is the matrix of the updating speed, $E=\left[\boldsymbol{e}_{1}, \boldsymbol{e}_{2}, \ldots, \boldsymbol{e}_{m} ; \dot{\boldsymbol{e}}_{1}, \dot{\boldsymbol{e}}_{2}, \ldots, \dot{\boldsymbol{e}}_{m}\right]^{T} \in$
$R^{m \times 2}$ is the system output error vector, and $\boldsymbol{P} \in R^{2 \times 1}$ is chosen matrix based on the stability condition in the sense of Lyapunov [28]. We adopted the following decoupled compensator to approximate the disturbances. The input of the fuzzy rule are $\boldsymbol{X}_{\boldsymbol{d}}, \dot{\boldsymbol{X}}_{\boldsymbol{d}}, \boldsymbol{X}$ and $\dot{\boldsymbol{X}}$. The output are the impedance force on each axis. To approximate the external disturbances:

$$
\begin{aligned}
\tau_{T_{2}} & =J_{T} \theta_{X}^{T} S(X) \\
\tau_{N_{12}} & =\left(I-J_{T}^{T}(q) J_{T}(q)_{M}^{+}\right) J_{W} \theta_{N}^{T} S(N)
\end{aligned}
$$

where $\boldsymbol{E}_{T}=\left[\boldsymbol{X}-\boldsymbol{X}_{d} ; \dot{\boldsymbol{X}}-\dot{\boldsymbol{X}}_{d}\right]^{T} \in R^{3 \times 2}$ and $\boldsymbol{E}_{N}=\left[\boldsymbol{e}_{N 1} ; \dot{\boldsymbol{e}}_{N 1}\right]^{T} \in$ $R^{3 \times 2}$.

Fig. 6 shows the overview of proposed improved humanrobot collaborative control (IHRCC). The final input term is defined as:

$$
\tau_{d}=\tau_{T_{1}}+\tau_{T_{2}}+\tau_{N_{11}}+\tau_{N_{12}}+\tau_{N_{2}}
$$

4) System Description: Fig. 8 shows the system architecture. We controlled a serial robot (LWR4+, KUKA, Germany) through the joint torques interface provided by the Fast Research Interface (FRI) [29]. The cable-driven master manipulator (Sigma 7, Force Dimension, Switzerland) and the switch pedal [30] were used to implement a scaling scheme of 3D Cartesian position for teleoperation. An endoscopic camera HD and an ArUco maker board are used to create the virtual surgical tasks in augmented reality [31]. The interaction force on the RCM constraint between the surgical tool shaft and the abdominal wall is measured with a force sensor (M8128C6, SRI, China). The description of software system is reported in our previous work [6][8][13].

TABLE I

CONTROLLER PARAMETERS LIST

\begin{tabular}{|c|l|}
\hline Controller & Controller parameters \\
\hline & $\boldsymbol{K}_{\boldsymbol{X}}=\operatorname{diag}[3000,3000,3000]$ \\
DCAC & $\boldsymbol{D}_{\boldsymbol{X}}=\operatorname{diag}[30,30,30]$ \\
$\left(\boldsymbol{\tau}_{\boldsymbol{d}}=\boldsymbol{\tau}_{\boldsymbol{T}_{\mathbf{1}}}+\boldsymbol{\tau}_{\boldsymbol{N}_{\mathbf{1 1}}}\right)$ & $\boldsymbol{K}_{\boldsymbol{N}}=\operatorname{diag}[800,800,800]$ \\
& $\boldsymbol{D}_{\boldsymbol{N}}=\operatorname{diag}[10,10,10], \quad \lambda=0.5$ \\
\hline & $\boldsymbol{K}_{\boldsymbol{X}}=\operatorname{diag}[3000,3000,3000]$ \\
& $\mathbf{D}_{\mathbf{X}}=\operatorname{diag}[30,30,30]$ \\
& $\boldsymbol{K}_{\boldsymbol{N}}=\operatorname{diag}[800,800,800]$ \\
$\left(\boldsymbol{\tau}_{\boldsymbol{d}}=\boldsymbol{\tau}_{\boldsymbol{T}_{\mathbf{1}}}+\boldsymbol{\tau}_{\boldsymbol{N}_{\mathbf{1 1}}}\right.$ & $\boldsymbol{D}_{\boldsymbol{N}}=\operatorname{diag}[10,10,10], \quad \lambda=0.5$ \\
$\left.+\boldsymbol{\tau}_{\boldsymbol{T}_{\mathbf{2}}}+\boldsymbol{\tau}_{\boldsymbol{N}_{\mathbf{1 2}}}+\boldsymbol{\tau}_{\boldsymbol{N}_{\mathbf{2}}}\right)$ & $k_{0}=0.1, k_{\psi}=150.0, d_{\psi}=5.0$ \\
& $\boldsymbol{P}=\operatorname{diag}[14,2], \rho=0.06$ \\
& $\boldsymbol{\gamma}=\operatorname{diag}[0.3,0.3,0.3,0.03,0.03,0.03]$ \\
& $\boldsymbol{M}_{\boldsymbol{\Theta}}=[0.5,0.5,0.5,0.5,0.5,0.5]$ \\
\hline
\end{tabular}

\section{Experimental Demonstration}

We experimentally demonstrate the concept of improved human-robot collaborative control approach. One teleoperator (User 2) and one doctor (User 1) were enrolled. The whole set up procedure [8] is presented in Fig. 9. The constraint swivel motion and the RCM constraint are validated with physical interaction, while the surgical tip is teleoperated to track virtual trajectories without physical interaction. The tool shaft should always go through the RCM constraint on the patient phantom during the teleoperation. The robot arm is moved by user 1 in an allowed swivel area [8]. Virtual surgical task paths in 


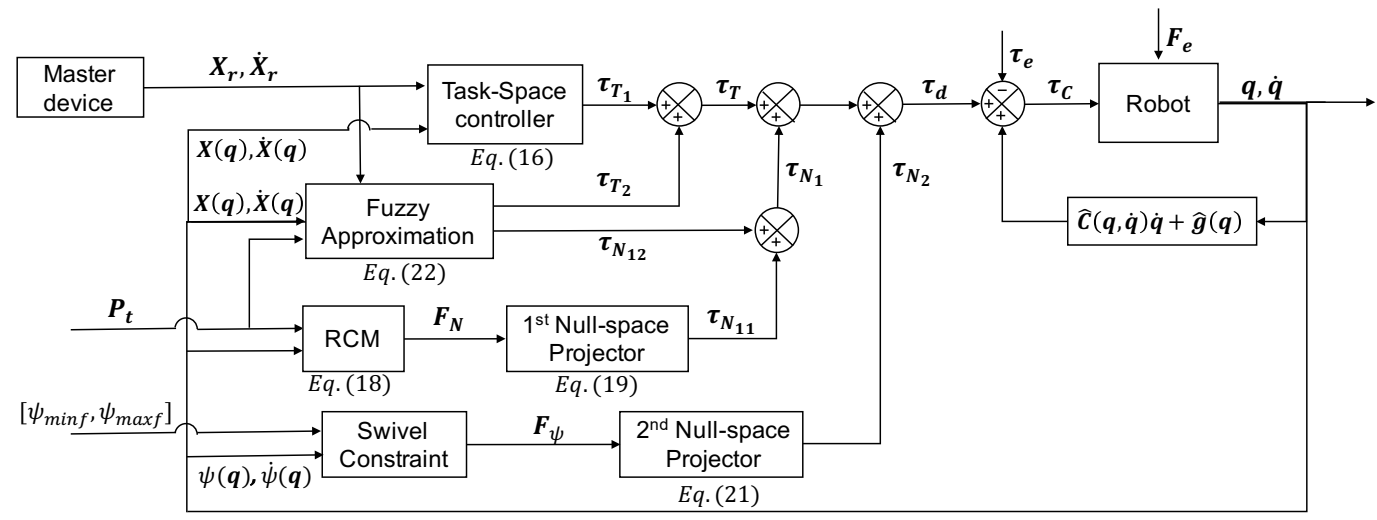

Fig. 6. Block diagram of the control architecture: The end-effector tracking is achieved by the "Task-space controller". The "RCM" block and the " 1 st Null-space Projector" are control term adopted to handle the RCM constraint, while the "Swivel Constraint" and the " $2^{\text {nd }}$ Null-space Projector" calculates the force applied to the elbow, and the "Fuzzy Approximation" compensates the unknown decoupled disturbance in the task space and to the RCM constraint. "Robot" represents robot arm dynamic model with uncertain physical interaction.

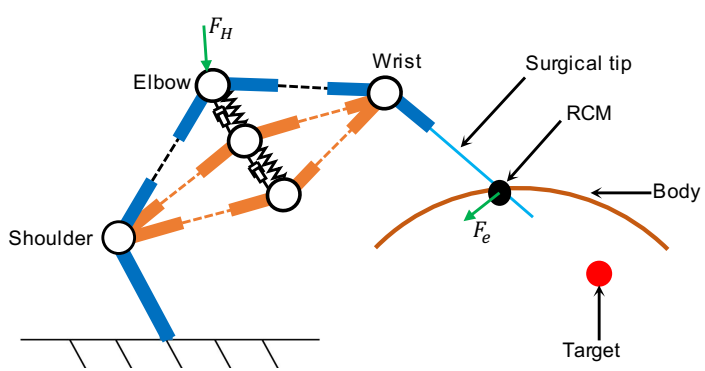

Fig. 7. Physical interaction and corresponding external disturbance: $\boldsymbol{F}_{\boldsymbol{H}}$ is the human hand force on the elbow, and $\boldsymbol{F}_{\boldsymbol{e}}$ is the force exerted on the surgical tool shaft generated from the physical interaction with the abdominal wall.

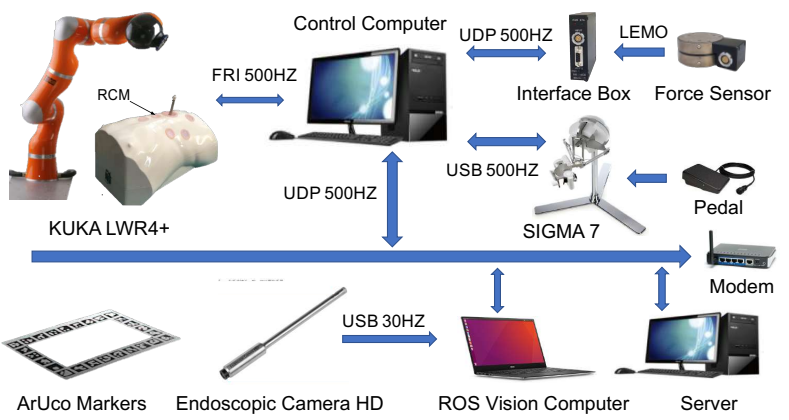

Fig. 8. Overview of the developed teleoperated surgical system.

the operational space were overlaid on the camera image [8]. The user 2 , sitting at the remote desk and using the monitor to observe the scene, is asked to follow the line with the robot end-effector. The performed trajectory is overlaid on the camera image plane. Table I shows the corresponding control parameters.

Fig. 10 shows the Cartesian position error, $\boldsymbol{E}_{X}$, the RCM constraint error, $d$, and the interaction force $\boldsymbol{F}_{\boldsymbol{e}}$. The magnitudes of them are recorded for performance comparison:

$$
\left\|E_{X}\right\|=\left\|X_{d}-X\right\|
$$
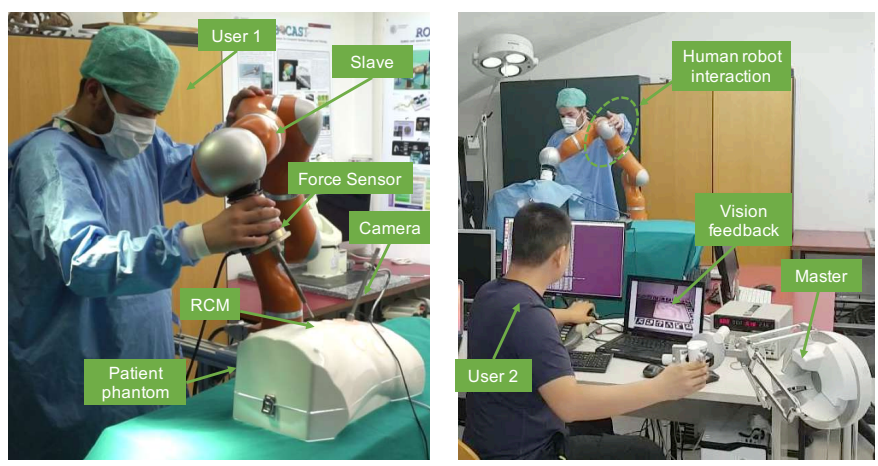

Fig. 9. Experimental setup procedures: (1) Firstly, hands-on control is utilized to allow user 1 locate the RCM constraint by hand on the patient phantom. (2) Secondly, teleoperated path tracking is activated in the following procedure. User 2 uses the master manipulator to drive the surgical tip tracking the medical tasks shown in augmented reality.

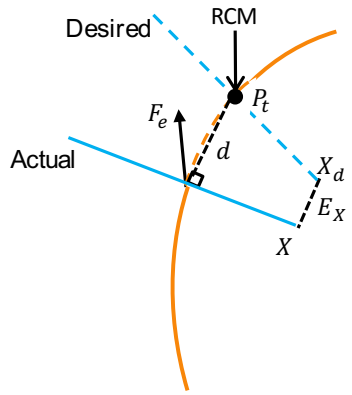

Fig. 10. Accuracy measurement. "Actual" represents the actual surgical tool shaft placement and "Desired" represents the desired surgical tool shaft placement.

$$
\begin{gathered}
d=\left\|\left(\boldsymbol{P}_{\boldsymbol{t}}-\boldsymbol{X}\right) \times \hat{\boldsymbol{u}}_{\boldsymbol{c}}\right\| \\
\left\|\boldsymbol{F}_{\boldsymbol{e}}\right\|=\sqrt{F_{x}^{2}+F_{y}^{2}+F_{z}^{2}}
\end{gathered}
$$

where $\hat{\boldsymbol{u}}_{\boldsymbol{c}}$ is the actual unit direction vector of the tip pose, $F_{x}, F_{y}$ and $F_{z}$ are the 3-axis force output of the force sensor. Firstly, comparison of performance between improved human- 
robot collaborative control strategy (IHRCC), $\tau_{d}=\tau_{T_{1}}+\tau_{T_{2}}+$ $\tau_{N_{11}}+\tau_{N_{12}}+\tau_{N_{2}}$, and decoupled Cartesian admittance control (DCAC) proposed by Sandoval et al. [5], $\tau_{d}=\tau_{T_{1}}+\tau_{N_{11}}$, are conducted with a fixed surgical tip position and a fixed RCM constraint. Secondly, the performance is compared with three different teleoperation tracking tasks. All the tracking tasks are repeated for 5 trials. Given the fixed surgical position

(a) Swivel motion

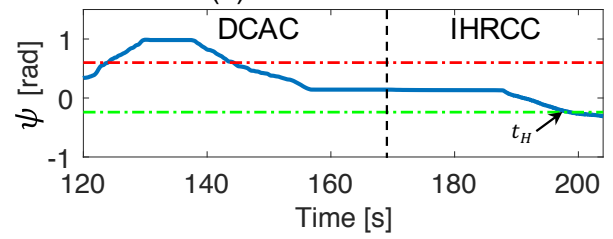

(b) Cartesian position error

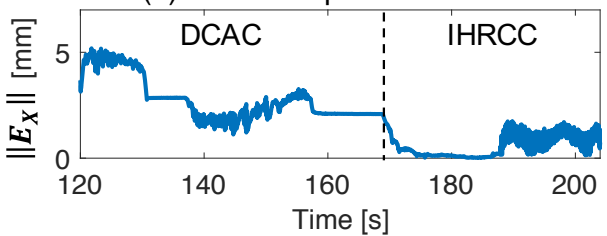

(c) RCM constraint error

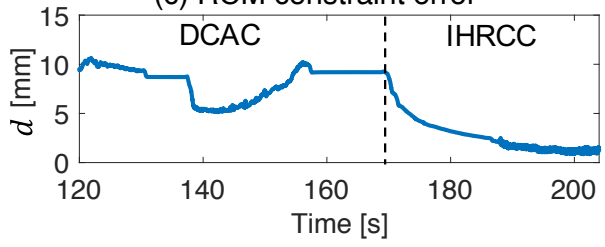

(d) Interaction force

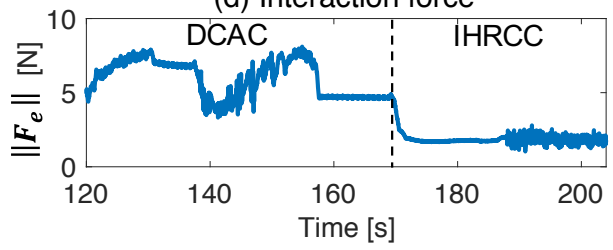

Fig. 11. Performance comparison with fixed pose between DCAC and IHRC. During human-guided swivel motion, hand force is applied by the human user on the robot arm but resisted by the robot from $t_{H}$.

and the fixed RCM constraint, the performance comparison is shown in Fig. 11. In the beginning, the DCAC controller was activated and user 1 moves the robot arm. Then the controller is switched to IHRCC. The swivel motion $\psi$ moved by user 1 is shown in Fig. 11(a). The corresponding Cartesian position error and the error of the RCM constraint are shown in Fig. 11(b) and Fig. 11(c). The magnitude of the interaction force $\boldsymbol{F}_{\boldsymbol{e}}$ on the abdominal wall is shown in Fig. 11(d). It is easy to see that the swivel boundary is constrained in the defined area $\left[\psi_{\min f}, \psi_{\max f}\right]$ by IHRCC. Moreover, according to the results, the Cartesian error and the error of the RCM constraint converges to a smaller range. Compared with the DCAC, $\left\|\boldsymbol{F}_{\boldsymbol{e}}\right\|$ is also constrained intro a small area.

The corresponding distribution of the Cartesian error, $\boldsymbol{E}_{\boldsymbol{X}}$, is shown in Fig. 12(a) and the corresponding distribution of RCM Constraint error $d$ is shown in Fig. 12(b). Since the virtual trajectory is in augmented reality, there is no interaction force on the surgical tip. The force is exerted on the abdominal wall by the tool shaft. Fig. 12(c) shows the corresponding distribution of interaction force $\left\|\boldsymbol{F}_{\boldsymbol{e}}\right\|$ between the abdominal wall and the tool shaft during the teleoperation tracking. Compared with DCAC, the accuracy of the surgical tip is improved, meanwhile the error of the RCM constraint and the interaction forces are converged into a smaller range.

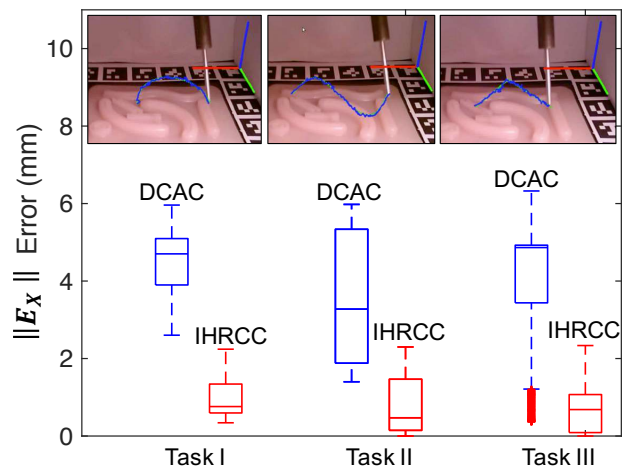

(a) Distribution of end-effector error $\left\|\boldsymbol{E}_{\boldsymbol{X}}\right\|$

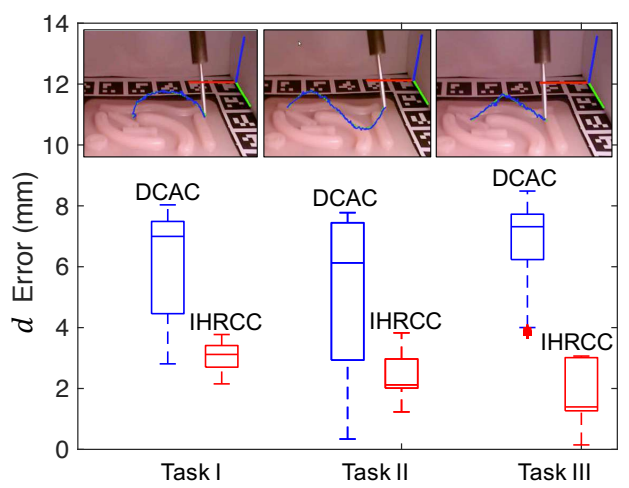

(b) Distribution of RCM constraint error $d$

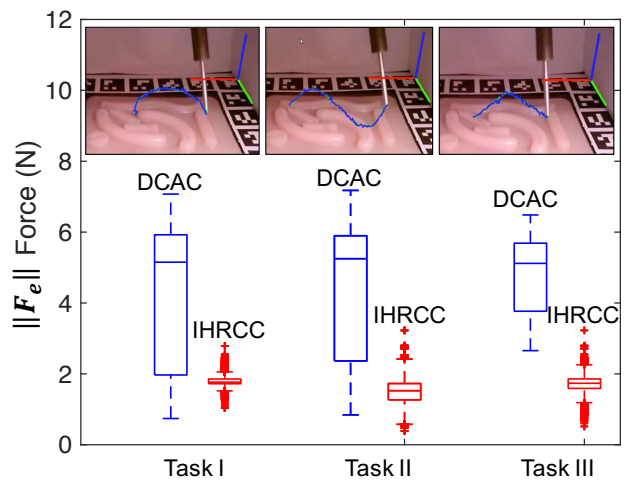

(c) Distribution of interaction force $\left\|\boldsymbol{F}_{\boldsymbol{e}}\right\|$ on the RCM constraint

Fig. 12. Comparison between DCAC and IHRCC.

\section{Discussion AND Conclusion}

A method of improved human-robot collaborative control is introduced for the teleoperated surgery in this paper. Ac- 
cording to the experimental results, the proposed algorithm (IHRCC) can achieve the surgical task respecting the RCM constraint and the compliant swivel motion in the defined safe area, without decreasing the accuracy. Moreover, the uncertain disturbances generated by the interaction from both the hand force and the physical contact force on the surgical tool shaft are compensated by the controller.

Compared to the method proposed by Sandoval et al. [5], the surgical task accuracy is improved, the RCM constraint and interaction force are constrained into a smaller area. Compared with our previous work [8][13], not only the surgical task accuracy and RCM constraint are improved, but also the computation efficiency is reinforced without trajectory planning. Surgeons can move the surgical arm during the ongoing task. The force imposed on the abdominal wall are decreased, which provides flexible workspace and lowers the risks of enlarging the small incision on the abdominal wall. The interaction force between the tool shaft and the abdominal wall around $1 \mathrm{~N}$ is acceptable without hurting the small incision [32]. The experimental evaluation in this paper is for a feasibility study of the proposed method with a proof of concept. In our future work, more subjects and a complete experimental validation will be conducted to verify its performance. A more sophisticated testing setup (adding one more force sensor for external forces sensing) will be conceived and performed with the physical interaction between the surgical tip and the organs).

\section{REFERENCES}

[1] N. Aghakhani, M. Geravand, N. Shahriari, M. Vendittelli, and G. Oriolo, "Task control with remote center of motion constraint for minimally invasive robotic surgery," in Robotics and Automation (ICRA), 2013 IEEE International Conference on. IEEE, 2013, pp. 5807-5812.

[2] H. Azimian, R. V. Patel, and M. D. Naish, "On constrained manipulation in robotics-assisted minimally invasive surgery," in Biomedical Robotics and Biomechatronics (BioRob), 2010 3rd IEEE RAS and EMBS International Conference on. IEEE, 2010, pp. 650-655.

[3] C.-H. Kuo and J. S. Dai, "Robotics for minimally invasive surgery: a historical review from the perspective of kinematics," in International symposium on history of machines and mechanisms. Springer, 2009, pp. 337-354.

[4] U. Hagn, R. Konietschke, A. Tobergte, M. Nickl, S. Jörg, B. Kübler, G. Passig, M. Gröger, F. Fröhlich, U. Seibold et al., "Dlr mirosurge: a versatile system for research in endoscopic telesurgery," International journal of computer assisted radiology and surgery, vol. 5, no. 2, pp. 183-193, 2010.

[5] J. Sandoval, G. Poisson, and P. Vieyres, "Improved dynamic formulation for decoupled cartesian admittance control and rcm constraint," in Robotics and Automation (ICRA), 2016 IEEE International Conference on. IEEE, 2016, pp. 1124-1129.

[6] H. Su, J. Sandoval, P. Vieyres, G. Poisson, G. Ferrigno, and E. De Momi, "Safety-enhanced collaborative framework for tele-operated minimally invasive surgery using a 7-dof torque-controlled robot," International Journal of Control, Automation and Systems, vol. 16, no. 6, pp. 29152923, 2018.

[7] T. Ortmaier and G. Hirzinger, "Cartesian control issues for minimally invasive robot surgery," in Intelligent Robots and Systems, 2000.(IROS 2000). Proceedings. 2000 IEEE/RSJ International Conference on, vol. 1. IEEE, 2000, pp. 565-571

[8] H. Su, J. Sandoval, M. R. Makhdoomi, G. Ferrigno, and E. De Momi, "Safety-enhanced human-robot interaction control of redundant robot for teleoperated minimally invasive surgery," in International Conference on Robotics and Automation, 2018, pp. 6611-6616.

[9] M. A. Peshkin, "Programmed compliance for error corrective assembly," IEEE Transactions on Robotics and Automation, vol. 6, no. 4, pp. 473482,1990
[10] F. Petit, A. Dietrich, and A. Albu-Schäffer, "Generalizing torque control concepts: using well-established torque control methods on variable stiffness robots," IEEE Robotics and Automation Magazine, vol. 22, no. 4, pp. 37-51, 2015.

[11] C. Yang, S. Amarjyoti, X. Wang, Z. Li, H. Ma, and C.-Y. Su, "Visual servoing control of baxter robot arms with obstacle avoidance using kinematic redundancy," in International Conference on Intelligent Robotics and Applications. Springer, 2015, pp. 568-580.

[12] H. Su, N. Enayati, L. Vantadori, A. Spinoglio, G. Ferrigno, and E. De Momi, "Online human-like redundancy optimization for teleoperated anthropomorphic manipulators," International Journal of Advanced Robotic Systems, vol. 15, no. 6, p. 1729881418814695, 2018.

[13] J. Sandoval, H. Su, P. Vieyres, G. Poisson, G. Ferrigno, and E. De Momi, "Collaborative framework for robot-assisted minimally invasive surgery using a 7-dof anthropomorphic robot," Robotics and Autonomous Systems, vol. 106, pp. 95-106, 2018.

[14] H. Su, G. Ferrigno, and E. De Momi, "Adaptive decoupling control of a serial redundant robot for teleoperated minimally invasive surgery," in IEEE ICRA Workshop on Supervised Autonomy in Surgical Robotics, 2018.

[15] Z. Li, C.-Y. Su, G. Li, and H. Su, "Fuzzy approximation-based adaptive backstepping control of an exoskeleton for human upper limbs," IEEE Transactions on Fuzzy Systems, vol. 23, no. 3, pp. 555-566, 2015.

[16] A. M. Dietrich, "Whole-body impedance control of wheeled humanoid robots," Ph.D. dissertation, Technische Universität München, 2015.

[17] A. Dietrich, C. Ott, and J. Park, "The hierarchical operational space formulation: Stability analysis for the regulation case," IEEE Robotics and Automation Letters, vol. 3, no. 2, pp. 1120-1127, 2018.

[18] L. Sciavicco and B. Siciliano, Modelling and control of robot manipulators. Springer Science \& Business Media, 2012.

[19] O. Khatib, "A unified approach for motion and force control of robot manipulators: The operational space formulation," IEEE Journal on Robotics and Automation, vol. 3, no. 1, pp. 43-53, 1987.

[20] C. Ott, "Cartesian impedance control: The rigid body case," in Cartesian Impedance Control of Redundant and Flexible-Joint Robots. Springer, 2008, pp. 29-44.

[21] J. Baillieul, "Kinematic programming alternatives for redundant manipulators," in Robotics and Automation. Proceedings. 1985 IEEE International Conference on, vol. 2. IEEE, 1985, pp. 722-728.

[22] A. Dietrich, Whole-body impedance control of wheeled humanoid robots. Springer, 2016

[23] L. Jin, S. Li, H. M. La, and X. Luo, "Manipulability optimization of redundant manipulators using dynamic neural networks," IEEE Transactions on Industrial Electronics, vol. 64, no. 6, pp. 4710-4720, June 2017.

[24] M. Shimizu, H. Kakuya, W.-K. Yoon, K. Kitagaki, and K. Kosuge, "Analytical inverse kinematic computation for 7-dof redundant manipulators with joint limits and its application to redundancy resolution," IEEE Transactions on Robotics, vol. 24, no. 5, pp. 1131-1142, 2008.

[25] J. Humphrey and C. Taylor, "Intracranial and abdominal aortic aneurysms: similarities, differences, and need for a new class of computational models," Annu. Rev. Biomed. Eng., vol. 10, pp. 221-246, 2008.

[26] E. Peña, B. Hernández-Gascón, and B. Calvo, "Human abdomen: Mechanical modeling and clinical applications," in Biomechanics of Living Organs. Elsevier, 2017, pp. 267-285.

[27] S. Tong and Y. Li, "Adaptive fuzzy output feedback control of mimo nonlinear systems with unknown dead-zone inputs," IEEE Transactions on Fuzzy Systems, vol. 21, no. 1, pp. 134-146, 2013.

[28] L.-X. Wang, "Stable adaptive fuzzy control of nonlinear systems," IEEE Transactions on fuzzy systems, vol. 1, no. 2, pp. 146-155, 1993.

[29] G. Schreiber, A. Stemmer, and R. Bischoff, "The fast research interface for the kuka lightweight robot," in IEEE Workshop on Innovative Robot Control Architectures for Demanding (Research) Applications How to Modify and Enhance Commercial Controllers (ICRA 2010), 2010, pp. $15-21$.

[30] N. Enayati, G. Ferrigno, and E. De Momi, "Skill-based human-robot cooperation in tele-operated path tracking," Autonomous Robots, pp. 113, 2018.

[31] N. Enayati, A. M. Okamura, A. Mariani, E. Pellegrini, M. M. Coad, G. Ferrigno, and E. De Momi, "Robotic assistance-as-needed for enhanced visuomotor learning in surgical robotics training: An experimental study," in 2018 IEEE International Conference on Robotics and Automation (ICRA). IEEE, 2018, pp. 6631-6636.

[32] B. Böhm, M. Knigge, M. Kraft, K. Gründel, and U. Boenick, "Influence of different trocar tips on abdominal wall penetration during laparoscopy," Surgical endoscopy, vol. 12, no. 12, pp. 1434-1438, 1998. 